\title{
DEFORMATIONS OF HOMOTOPY ALGEBRAS
}

\author{
VLADIMIR HINICH
}

\section{INTRODUCTION}

1.1. Let $k$ be a field of characteristic zero, $\mathcal{O}$ be a dg operad over $k$ and let $A$ be an $\mathcal{O}$-algebra. In this note we define formal deformations of $A$, construct the deformation functor

$$
\operatorname{Def}_{A}: \operatorname{dgart}^{\leq 0}(k) \rightarrow \Delta^{0} \text { Ens }
$$

from the category of artinian local dg algebras (see 1.5 for the precise definition) to the category of simplicial sets. In the case $\mathcal{O}$ and $A$ are non-positively graded, we prove that $\operatorname{Def}_{A}$ is governed by the tangent Lie algebra $T_{A}$ defined in [H2]. A very easy example 2.2 shows that the result does not hold without this condition.

1.2. "Classical" formal deformation theory over a field of characteristic zero can be described as follows.

Let $k$ be a field of characteristic zero, art $(k)$ be the category of artinian local $k$-algebras with residue field $k$. Let $\mathcal{C}$ be a category cofibred over art $(k)$. Equivalently, this means that a 2 -functor

$$
\mathcal{C}: \operatorname{art}(k) \rightarrow \text { Cat }
$$

is given, that is a collection of categories $\mathcal{C}(R), R \in \operatorname{art}(k)$, of functors $f^{*}: \mathcal{C}(R) \rightarrow \mathcal{C}(S)$ for each morphism $f: R \rightarrow S$ in $\operatorname{art}(k)$ and of isomorphisms $f^{*} g^{*} \stackrel{\sim}{\longrightarrow}(f g)^{*}$ satisfying the cocycle condition.

Finally, let an object $A \in \mathcal{C}(k)$ be given. Then the deformation functor

$$
\operatorname{Def}_{A}: \operatorname{art}(k) \rightarrow \operatorname{Grp}
$$

assigns to each $R \in \operatorname{art}(k)$ the groupoid whose objects are isomorphisms $\alpha: \pi^{*}(B) \rightarrow A$ where $\pi: R \rightarrow k$ is the natural map, and morphisms are isomorphisms $B \rightarrow B^{\prime}$ compatible with $\alpha$ and $\alpha^{\prime}$.

If we are lucky enough (and this is usually the case), one can naturally assign to $A$ a $\operatorname{dg}$ Lie algebra $\mathfrak{g}$ which governs deformation of $A$ in the following sense: there is a natural equivalence of groupoids

$$
\operatorname{Def}_{A} \rightarrow \operatorname{Del}_{\mathfrak{g}}
$$

as functors from $\operatorname{art}(k)$ to Grp, where $\operatorname{Del}_{\mathfrak{g}}$ is the Deligne groupoid of $\mathfrak{g}$ - see GM, H1].

As we argued in [H3], the existence of dg Lie algebra $\mathfrak{g}$ is equivalent to the existence of formal dg moduli - given by the dg coalgebra $\mathcal{C}(\mathfrak{g})$. This also means that the functor $\operatorname{Def}_{A}$ can be extended to a functor on the category dgart $\leq^{0}(k)$ of non-positively graded $\mathrm{dg}$ commutative artinian algebras with residue field $k$ with values in $\Delta^{0}$ Ens. 
1.3. The category of algebras over a given $\mathrm{dg}$ operad $\mathcal{O}$ is not just a category - there exist weak equivalences, homotopies and higher homotopies between the algebras. Therefore, the above described approach can not produce a reasonable definition of formal deformations of operad algebras.

Also, we want that deformations of quasi-isomorphic algebras be equivalent, as well as deformations of algebras over quasi-isomorphic operads, so we should use a sort of "derived" notion of the deformation groupoid.

\subsubsection{Morally, the picture should be the following.}

For each $R \in$ dgart $\leq^{0}(k)$ a $\infty$-category of $R \otimes \mathcal{O}$-algebras should be defined; denote it $\operatorname{Alg}^{\infty}(\mathcal{O}, R)$. The collection of $\operatorname{Alg}^{\infty}(\mathcal{O}, R)$ should form an $\infty$-category cofibred over dgart $\leq 0(k)$.

Let now $A \in \operatorname{Alg}^{\infty}(\mathcal{O}, k)$. Then the deformation functor

$$
\operatorname{Def}_{A}: \operatorname{dgart}^{\leq 0}(k) \rightarrow \operatorname{Grp}^{\infty}
$$

should be a $(\infty-)$ functor to $\infty$-groupoids; its objects are $\infty$-isomorphisms $\alpha: \pi_{*}(B) \rightarrow A$ and morphisms $-\infty$-isomorphisms $B \rightarrow B^{\prime}$ commuting with $\alpha$ and $\alpha^{\prime}$.

1.3.2. Since we do not know well what an $\infty$-category is and how to assign an $\infty$-category to the category of operad algebras, we are looking for an appropriate substitute.

According to [H2], the category $\operatorname{Alg}(\mathcal{O}, R)$ of $R \otimes \mathcal{O}$-algebras admits a simplicial closed model category (SCMC) structure.

As a substitute to the $\infty$-category $\operatorname{Alg}^{\infty}(\mathcal{O}, R)$, we suggest the simplicial category $\operatorname{Alg}_{*}^{c}(\mathcal{O}, R)$ of cofibrant $R \otimes \mathcal{O}$-algebras.

This allows us to define a deformation groupoid as a functor

$$
\operatorname{Def}_{A}: \operatorname{dgart}^{\leq 0}(k) \rightarrow \Delta^{0} \text { Ens }
$$

to the simplicial sets.

Now, according to the general philosophy of deformation theory, the functor $\operatorname{Def}_{A}$ should be equivalent to the nerve of a certain dg Lie algebra $\mathfrak{g}$ - see H3], Sect. 8 - which is "responsible" for the deformations of $A$. In [H2], Sect. 7, we constructed a functorial tangent dg Lie algebra $T_{A} \in \operatorname{dglie}(k)$ as the Lie algebra $\operatorname{Der}(\widetilde{A}, \widetilde{A})$ of derivations of a cofibrant resolution $\widetilde{A}$ of $A$. The main result of this paper says that $T_{A}$ governs the formal deformations of $A$ provided $\mathcal{O}$ and $A$ belong to $C^{\leq 0}(k)$. Quite unexpectedly, there is a very simple counter-example showing that the last condition is necessary — see Example 2.2.

1.4. Content of the Sections. Throughout the paper we work a lot with simplicial categories and simplicial groupoids. We collect in the Appendix the necessary information about the subject. It is mostly well-known or easily imaginable; thus, the closed model category structure on simplicial categories is a slight generalization of a result of [DK].

In Section 2 we construct the deformation functor (1). To compare it with the nerve of the tangent dg Lie algebra, we provide in Section 3 a version of the nerve construction of [H3], Sect. 8, which assigns to a $\mathrm{dg}$ Lie algebra $\mathfrak{g}$ and to a $\operatorname{dg}$ artinian algebra $R$ a simplicial groupoid. 
Finally, in Section 1 we prove the main result. It follows easily from the model category structure of the category of simplicial categories.

1.5. Notation. In what follows we use the following notations for different categories.

Ens, Grp, Cat are the categories of sets, small groupoids and small categories respectively.

$\Delta$ is the category of ordered sets $[n]=\{0, \ldots, n\}, n \geq 0$ and order-preserving maps. For a category $\mathcal{C}$ we denote by $\Delta^{0} \mathcal{C}$ the category of simplicial objects in $\mathcal{C}$.

A simplicial category (and, in particular, a simplicial groupoid) will be supposed to have a discrete set of objects, if it is not explicitly specified otherwise. The category of small simplicial categories is defined sCat and that of simplicial groupoids sGrp.

For a fixed field $k$ of characteristic zero $\operatorname{dglie}(k)$ is the category of dg Lie algebras and dgart $\leq 0(k)$ is the category of non-positively graded commutative artinian dg algebras with residue field $k$.

1.6. Acknowledgement. This work was made during my stay at the Max-Planck Institut für Mathematik at Bonn. I express my gratitude to the Institute for the hospitality.

\section{Homotopy Algebras}

In this Section we define deformations of dg operad algebras.

Let $k$ be a fixed field of characteristic zero, $\mathcal{O} \in \mathrm{Op}(C(k))$ be a dg operad over $k, A \in \mathrm{Alg}(\mathcal{O})$ be a $\mathcal{O}$-algebra.

Let $R$ be a commutative dg $k$-algebra. Componentwise tensoring by $R$ defines a functor on $\mathrm{Op}(C(k))$ (its values can be equally considered in $\mathrm{Op}(C(k))$ and in $\mathrm{Op}(\bmod (R)))$. We define by $\operatorname{Alg}(\mathcal{O}, R)$ the category of $R \otimes \mathcal{O}$-algebras. This category admits a CMC structure - see [H2] with quasi-isomorphisms as weak equivalences and surjective maps as fibrations. We denote by $\operatorname{Alg}^{c}(\mathcal{O}, R)$ the full subcategory of cofibrant algebras and by $\mathcal{W}^{c}(\mathcal{O}, R)$ the category of cofibrant $\mathcal{O}$ - $R$-algebras with weak equivalences as arrows.

The category $\operatorname{Alg}(\mathcal{O})$ admits also a simplicial structure so that Quillen's axiom (SM7) is satisfied - see [H2], 4.8. The simplicial structure is defined by the simplicial path functor which assigns to an algebra $A \in \operatorname{Alg}(\mathcal{O})$ and to a finite simplicial set $S \in \Delta^{0}$ Ens the algebra $A^{S}=\Omega(S) \otimes A$ where $\Omega(S)$ denotes the dg commutative algebra of polynomial differential forms on $S$.

In the sequel we will add asterisk to denote that we consider the corresponding simplicial category. Thus, for example, $\mathcal{W}_{*}^{c}(\mathcal{O}, R)$ is the simplicial category whose objects are cofibrant $R \otimes \mathcal{O}$ algebras and whose $n$-morphisms from $x$ to $y$ consist of quasi-isomorphisms $x \rightarrow \Omega\left(\Delta^{n}\right) \otimes y$.

Recall [H3] that it is worthwhile to consider artinian local non-positively graded $\mathrm{dg} k$-algebras $(R, \mathfrak{m}) \in \operatorname{dgart}^{\leq 0}(k)$ as bases of formal deformations.

2.1. Definition. Let $A \in \operatorname{Alg}(\mathcal{O})$. Deformation functor

$$
\operatorname{Def}_{A}: \operatorname{dgart} \leq 0(k) \rightarrow \Delta^{0} \text { Ens }
$$


is defined by the formula

$$
\operatorname{Def}_{A}(R)=\mathrm{h}-\operatorname{fib}_{A}\left(\mathcal{N}\left(\mathcal{W}_{*}^{c}(\mathcal{O}, R)\right) \rightarrow \mathcal{N}\left(\mathcal{W}_{*}^{c}(\mathcal{O}, k)\right)\right)
$$

Here $\mathcal{N}:$ sCat $\rightarrow \Delta^{0}$ Ens is the simplicial nerve functor (see 5.3.1) and the homotopy fiber h-fib being taken at a point $\widetilde{A} \in \mathcal{W}_{*}^{c}(\mathcal{O}, k)$ where $\widetilde{A} \rightarrow A$ is a cofibrant resolution of $A$.

2.1.1. Recall (see [H2]) that for a $\mathcal{O}$-algebra $A$ its tangent Lie algebra $T_{A}$ is defined as

$$
T_{A}=\operatorname{Der}(\widetilde{A}, \widetilde{A}) .
$$

Now we are ready to formulate the main result of this note.

2.1.2. Theorem. Let $\mathcal{O}$ be a $d g$ operad over a field $k$ of characteristic zero and let $A$ be an $\mathcal{O}$-algebra. Suppose that both $\mathcal{O}$ and $A$ are non-positively graded. Then the deformation functor $\operatorname{Def}_{A}: \operatorname{dgart} \leq 0(k) \rightarrow \Delta^{0}$ Ens is equivalent to the nerve $\Sigma_{\mathfrak{g}}$ of the tangent $d g$ Lie algebra $\mathfrak{g}:=T_{A}$.

Theorem 2.1.2 will be proven in Section 4 . Now we give an elementary example which shows that the non-positivity condition is necessary.

2.2. Example. Let $\mathcal{O}$ be the trivial operad $\mathcal{O}(1)=k \cdot 1, \mathcal{O}(i)=0$ for $i \neq 0$. $\mathcal{O}$-algebras are just complexes and derivations are just all endomorphisms.

Let $A$ be the complex with zero differential with $A^{i}=k$ for all $i \in \mathbb{Z}$.

Then $T_{A}=\operatorname{Hom}(A, A)$ is a complex with zero differential; an element $f \in\left(\mathfrak{m} \otimes T_{A}\right)^{1}$ satisfies the Maurer-Cartan equation iff $f^{2}=0$. For instance, put $R=k[\epsilon] / \epsilon^{2}$. Then any element $f$ of degree one is Maurer-Cartan. The corresponding to $f$ complex of $R$-modules is $R \otimes A$ as a graded $R$-module, and has $f$ as the differential. Suppose that all components of $f$ are non-zero. Then $(R \otimes A, f)$ is contractible and of course can not be thought of being a deformation of $A$.

\section{Simplicial Deligne groupoid}

3.1. Definition. Let $k$ be a field of characteristic zero and $\mathfrak{g} \in \operatorname{dglie}(k)$ be a nilpotent $\mathrm{dg}$ Lie $k$-algebra. In this Section we construct a simplicial groupoid $\Gamma(\mathfrak{g})=\left\{\Gamma_{n}(\mathfrak{g})\right\}$ whose nerve (see 5.3.1) is naturally homotopically equivalent to the nerve $\Sigma(\mathfrak{g})$.

The construction is a generalization (and a simplification) of the one we used in [H3], 9.7.6.

Recall (see [H1], [H3], 8.1.1) that the nerve $\Sigma(\mathfrak{g})$ of the nilpotent dg Lie algebra $\mathfrak{g}$ is defined as

$$
\Sigma_{n}(\mathfrak{g})=\mathrm{MC}\left(\Omega_{n} \otimes \mathfrak{g}\right),
$$

$\Omega_{n}$ being the algebra of polynomial differential forms on the standard $n$-simplex.

Following [H3], Sect. 8, define a simplicial group $G=G(\mathfrak{g})$ by the formula

$$
G_{n}=\exp \left(\Omega_{n} \otimes \mathfrak{g}\right)^{0} .
$$

Here $\Omega_{n} \otimes \mathfrak{g}$ is a nilpotent dg Lie algebra, so its zero component is an honest nilpotent Lie algebra, and therefore its exponent makes sense. 
Define a simplicial groupoid $\Gamma:=\Gamma(\mathfrak{g})$ (we will call it simplicial Deligne groupoid since its zero component is the conventional Deligne groupoid [GM]) as follows.

$\mathrm{Ob} \Gamma=\mathrm{MC}(\mathfrak{g})$;

$\operatorname{Hom}_{\Gamma}(x, y)_{n}=\left\{g \in G_{n} \mid g(x)=y\right\}$.

It is useful to have in mind the following easy

3.1.1. Lemma. The simplicial group $G(\mathfrak{g})$ is always contractible.

Proof. As a simplicial set, $G$ is isomorphic to the simplicial vector space

$$
n \mapsto\left(\Omega_{n} \otimes \mathfrak{g}\right)^{0} .
$$

The latter is a direct sum of simplicial vector spaces of form $\Omega_{\bullet}^{p}$ (each one $\operatorname{dim} \mathfrak{g}^{-p}$ times) which are all contractible - see [], p. 44.

3.2. Equivalence. Recall that any simplicial category (and more generally, any $\mathcal{C} \in \Delta^{0}$ Cat) defines a bisimplicial set whose diagonal is called the nerve of $\mathcal{C}$, denoted by $\mathcal{N}(\mathcal{C})$ - see 5.3.1.

3.2.1. Proposition. The nerve $\Sigma(\mathfrak{g})$ of a nilpotent $d g$ Lie algebra is naturally homotopically equivalent to $\mathcal{N}(\Gamma(\mathfrak{g}))$.

Proof. Define $\Gamma^{\prime} \in \Delta^{0} \mathrm{Grp}$ (a simplicial groupoid in the wide sense) by the following formulas.

$\mathrm{Ob} \Gamma_{n}^{\prime}=\mathrm{MC}\left(\Omega_{n} \otimes \mathfrak{g}\right)$

$\operatorname{Hom}_{\Gamma^{\prime}}(x, y)_{n}=\left\{g \in G_{n}(\mathfrak{g}) \mid g(x)=y\right\}$.

One has a natural fully faithful embedding $\Gamma(\mathfrak{g}) \rightarrow \Gamma^{\prime}$. According to [H3], 8.2.5, the map $\Gamma_{n}(\mathfrak{g}) \rightarrow \Gamma_{n}^{\prime}$ is an equivalence of groupoids for each $n$. This implies that the induced map of the nerves

$$
\mathcal{N}(\Gamma) \rightarrow \mathcal{N}\left(\Gamma^{\prime}\right)
$$

is a homotopy equivalence.

Now we shall compare the nerve $\mathcal{N}\left(\Gamma^{\prime}\right)$ to $\Sigma(\mathfrak{g})$. Look at $\Gamma^{\prime}$ as at a bisimplicial set. One has

$$
\Gamma_{p q}^{\prime}=\Sigma_{p}(\mathfrak{g}) \times G_{p}(\mathfrak{g})^{q} .
$$

This means that the simplicial set $\Gamma_{\bullet q}^{\prime}$ is equal to $\Sigma(\mathfrak{g}) \times G(\mathfrak{g})^{q}$.

The simplicial set $G(\mathfrak{g})$ is contractible by Lemma 3.1.1. Therefore, $\Gamma_{\bullet q}^{\prime}$ is canonically homotopy equivalent to $\Sigma(\mathfrak{g})$. This implies that the nerve $\mathcal{N}\left(\Gamma^{\prime}\right)$ is homotopy equivalent to $\Sigma(\mathfrak{g})$.

3.2.2. Remark. Proposition 3.2.1 generalizes the claim used in the proof of 9.7.6 of [H3]. 
3.2.3. Let now $\mathfrak{g} \in \operatorname{dglie}(k)$. Following the well-known pattern, we define the functor

$$
\Gamma_{\mathfrak{g}}: \operatorname{dgart} \leq 0(k) \rightarrow \mathrm{sGrp}
$$

by the formula

$$
\Gamma_{\mathfrak{g}}(R)=\Gamma(\mathfrak{m} \otimes \mathfrak{g})
$$

for $(R, \mathfrak{m}) \in \operatorname{dgart} \leq 0(k)$.

The functor $\Gamma_{\mathfrak{g}}$ is also called the simplicial Deligne groupoid.

3.3. Properties. We wish to deduce now some properties of the simplicial Deligne groupoid functor which are similar to the properties of the nerve $\Sigma(\mathfrak{g})$ - see [H3], Sect. 8.

In what follows we use the closed model category (CMC) structure on the category sCat see 5.1.

3.3.1. Proposition. Let $f: \mathfrak{g} \rightarrow \mathfrak{h}$ be surjective (resp., a surjective quasi-isomorphism). Then for each $(R, \mathfrak{m}) \in \operatorname{dgart} \leq 0(k)$ the map

$$
f: \Gamma_{\mathfrak{g}}(R) \rightarrow \Gamma_{\mathfrak{h}}(R)
$$

is a fibration (resp., an acyclic fibration) in sCat.

Proof. Note first of all that the similar claim holds for the nerve functor: according to [H3], Prop. 7.2.1, the map $f: \Sigma_{\mathfrak{g}}(R) \rightarrow \Sigma_{\mathfrak{h}}(R)$ is a fibration (resp., acyclic fibration) provided $f$ is a surjection (resp., a surjective quasi-isomorphism). This implies that the map

$$
f: \Gamma_{\mathfrak{g}}(R) \rightarrow \Gamma_{\mathfrak{h}}(R)
$$

satisfiest the property (1) of fibrations (resp., of acyclic fibrations) - see 5.1.4, 5.1.7.

Let us check the property (2). It claims that for any $x, y \in \mathrm{Ob} \Gamma_{\mathfrak{g}}(R)$ the map of simplicial sets

$$
f: \mathcal{H o m}_{\mathfrak{g}}(x, y) \rightarrow \mathcal{H o m}_{\mathfrak{h}}(f x, f y)
$$

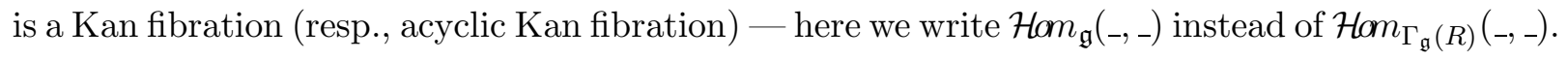
Denote $G=G(\mathfrak{g}), H=G(\mathfrak{h})$ the simplicial groups corresponding to $\mathfrak{g}, \mathfrak{h}$ as in the formula $\sharp$.

A map from a simplicial set $K$ to $\mathcal{H}_{\mathfrak{g}}(x, y)$ is given by an element $g \in G(K)=\operatorname{Hom}(K, G)$ satisfying the condition $g(x)=y$.

Let a commutative diagram in $\Delta^{0}$ Ens

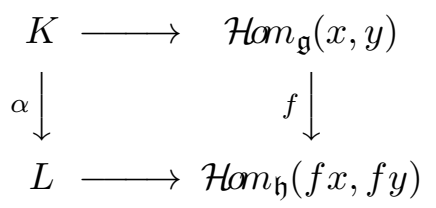


be given with $\alpha: K \rightarrow L$ being a cofibration of finite simplicial sets. We suppose also that either $\alpha$ or $f$ is a weak equivalence. Our aim is to find a map $L \rightarrow \mathcal{H o m}_{\mathfrak{g}}(x, y)$ commuting with the above diagram. Thus, we are given with a compatible pair of elements $g \in G(K), h \in H(L)$ satisfying the groperty

$$
g(x)=y ; h(f x)=f y .
$$

Our aim is to lift this pair to an element $\widetilde{g} \in G(L)$ satisfying the property $\widetilde{g}(x)=y$.

We will do this in two steps. First of all, since $f$ is surjective, the induced map of simplicial groups $f: G \rightarrow H$ is surjective, and, therefore, fibrant. Furthermore, since both $G$ and $H$ are contractible by Lemma 3.1.1, the map $f: G \rightarrow H$ is actually an acyclic fibration, and therefore the pair of compatible elements $g \in G(K), h \in H(L)$ lifts to an element $g^{\prime} \in G(L)$. We can not, unfortunately, be sure that $g^{\prime}(x)=y$. This is why we need the second step which will correct $g^{\prime}$ to satisfy this property.

Suppose $g^{\prime}(x)=y^{\prime} \in \operatorname{MC}(\Omega(L) \otimes \mathfrak{m} \otimes \mathfrak{g})$. The elements $y$ and $y^{\prime}$ of $\operatorname{MC}(\Omega(L) \otimes \mathfrak{m} \otimes \mathfrak{g})$ have the same images in both $\mathrm{MC}(\Omega(K) \otimes \mathfrak{m} \otimes \mathfrak{g})$ and $\mathrm{MC}(\Omega(L) \otimes \mathfrak{m} \otimes \mathfrak{h})$. Now, the commutative diagram

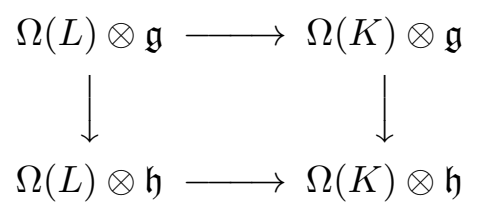

induces an acyclic fibration

$$
p: \mathfrak{g}_{1}:=\Omega(L) \otimes \mathfrak{g} \rightarrow \Omega(K) \otimes \mathfrak{g} \times \Omega(K) \otimes \mathfrak{h}(L) \otimes \mathfrak{h}=: \mathfrak{g}_{2}
$$

of dg Lie algebras. Then the map $\Sigma_{p}: \Sigma_{\mathfrak{g}_{1}}(R) \rightarrow \Sigma_{\mathfrak{g}_{2}}(R)$ is an acyclic fibration.

Now, we have two elements $y, y^{\prime} \in \mathrm{MC}\left(\mathfrak{m} \otimes \mathfrak{g}_{1}\right)$ satisfying $p(y)=p\left(y^{\prime}\right) \in \mathrm{MC}\left(\mathfrak{m} \otimes \mathfrak{g}_{2}\right)$. Therefore, there exists an element $z \in \Sigma_{\mathfrak{g}_{1}}(R)_{1}$ such that $d_{0} z=y, d_{1} z=y^{\prime}$ and $p(z)=s_{0}(p(y))$. Using the explicit description of $\Sigma_{\mathfrak{g}}(R)_{1}$ in [H3], 8.2.3, one obtains and element $\gamma \in \exp \left(\mathfrak{m} \otimes \mathfrak{g}_{1}\right)$ satisfying $p(\gamma)=1 \in \exp \left(\mathfrak{m} \otimes \mathfrak{g}_{2}\right) ; \gamma\left(y^{\prime}\right)=y$.

Then one immediately sees that the element $\widetilde{g}=\gamma g^{\prime}$ is the one we need.

3.3.2. Corollary. 1. For any $\mathfrak{g} \in \operatorname{dglie}(k), R \in \operatorname{dgart} \leq 0(k), x, y \in \mathrm{Ob}_{\mathfrak{g}}(R)$ the simplicial set $\mathcal{H o m}(x, y)$ is fibrant.

2. Any quasi-isomorphism $f: \mathfrak{g} \rightarrow \mathfrak{h}$ induces a weak equivalence

$$
f: \Gamma_{\mathfrak{g}}(R) \rightarrow \Gamma_{\mathfrak{h}}(R)
$$

for each $R \in \operatorname{dgart} \leq 0(k)$.

Proof. 1. Take $\mathfrak{h}=0$ in Proposition 3.3.1.

2. The category $\operatorname{dglie}(k)$ admits a CMC structure with surjections as fibrations and quasiisomorphisms as weak equivalences - see [H2], Sect. 4. Using this, present $f=p \circ i$ as a composition of an acyclic fibration $p$ and an acyclic cofibration $i$. Any acyclic cofibration in 
$\operatorname{dglie}(k)$ is left invertible: $q \circ i=\mathrm{id}$. The map $q$ is obviously an acyclic fibration. Then by Proposition 3.3.1 the map $f: \Gamma_{\mathfrak{g}}(R) \rightarrow \Gamma_{\mathfrak{h}}(R)$ is a weak equivalence.

\section{FinAL}

4.1. We start with an observation explaining the connection between $T_{A}$ and the formal deformations of $A$. Let $B$ be a cofibrant $R \otimes \mathcal{O}$-algebra with $(R, \mathfrak{m}) \in \operatorname{dgart} \leq^{\leq 0}(k)$. Denote $A=k \otimes_{R} B$. The algebra $B$ is isomorphic, as a graded $\mathcal{O}$-algebra, to $R \otimes A$. Choose a graded isomorphism

$$
\theta: B \rightarrow R \otimes A
$$

and put

$$
z=\theta \circ d_{B} \circ \theta^{-1}-1 \otimes d_{A}
$$

where $d_{B}$ (resp., $\left.d_{A}\right)$ is the differential in $B$ (resp., in $A$ ).

Then $z$ is a degree one derivation in $\mathfrak{m} \otimes T_{A}$ satisfying the Maurer-Cartan equation. A different choice of isomorphism $\theta$ gives rise to a Maurer-Cartan element $z^{\prime} \in \mathfrak{m} \otimes T_{A}$ equivalent to $z$ : there exists $g \in \exp \left(\mathfrak{m} \otimes T_{A}\right)^{0}$ such that $z^{\prime}=g(z)$.

In what follows we will use a (non-unique) presentation of a $R \otimes \mathcal{O}$-algebra $B$ by an element $z \in \mathrm{MC}\left(\mathfrak{m} \otimes T_{k \otimes_{R} B}\right)$.

4.2. Proof of the Theorem. To simplify the notation, denote $\mathcal{W}=\mathcal{W}_{*}^{c}(\mathcal{O}, R), \overline{\mathcal{W}}=\mathcal{W}_{*}^{c}(\mathcal{O}, k)$.

4.2.1. Lemma. The natural map $\pi: W \rightarrow \bar{W}$ is a fibration in sCat.

Proof. 1. Let us prove the condition (1) of Definition 5.1.4.

It means the following. Let $f: A \rightarrow B$ be a quasi-isomorphism of cofibrant $\mathcal{O}$-algebras over $k$. Let one of two elements $a \in \mathrm{MC}\left(\mathfrak{m} \otimes T_{A}\right)$ or $b \in \mathrm{MC}\left(\mathfrak{m} \otimes T_{B}\right)$ be given. We have to check that there exists a choice of the second element and a map

$$
g:(R \otimes A, d+a) \rightarrow(R \otimes B, d+b)
$$

of $R \otimes \mathcal{O}$-algebras which lifts $f: A \rightarrow B$.

We can consider separately the cases when $f$ is an acyclic fibration or an acyclic cofibration.

In both cases we will be looking for the map $g$ in the form

$$
g=\gamma_{B}^{-1} \circ\left(\mathrm{id}_{R} \otimes f\right) \circ \gamma_{A}
$$

where $\gamma_{A} \in \exp \left(\mathfrak{m} \otimes T_{A}\right)^{0}$ and similarly for $\gamma_{B}$. A map $g$ as above should commute with the differentials $d+a$ and $d+b$. This amounts to the condition

$$
f_{*}\left(\gamma_{A}(a)\right)=f^{*}\left(\gamma_{B}(b)\right),
$$

where the natural maps

$$
T_{A} \stackrel{f_{*}}{\longrightarrow} \operatorname{Der}_{f}(A, B) \stackrel{f^{*}}{\longleftarrow} T_{B}
$$

are defined as in [H2], 8.1.

Recall that we are assuming that $f$ is either acyclic cofibration or an acyclic fibration.

In both cases there exists a commutative square 


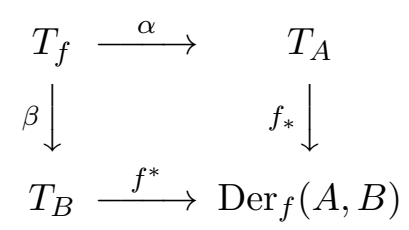

where $T_{f}$ is a dg Lie algebra and $\alpha, \beta$ are Lie algebra quasi-isomorphisms - see [H2], 8.2, 8.3. The maps $\alpha, \beta$ induce bijections

$$
\pi_{0}\left(\Sigma_{T_{A}}(R)\right) \longleftarrow \pi_{0}\left(\Sigma_{T_{f}}(R)\right) \longrightarrow \pi_{0}\left(\Sigma_{T_{A}}(R)\right)
$$

which prove the assertion.

2. Let us check the condition (2) of 5.1.4. Let $\widetilde{A}, \widetilde{B} \in \mathcal{W}$ and let $A=k \otimes_{R} \widetilde{A}, B=k \otimes_{R} \widetilde{B}$. We have to check that the map

$$
\mathcal{H o m}(\widetilde{A}, \widetilde{B}) \rightarrow \mathcal{H o m}(A, B)
$$

is a Kan fibration. But this results from the $\mathrm{SCMC}$ structure on $\operatorname{Alg}(R \otimes \mathcal{O})$. In fact, $\widetilde{A}$ is cofibrant, and the reduction map $\widetilde{B} \rightarrow B$ can be considered as a fibration in $\operatorname{Alg}(R \otimes \mathcal{O})$. Therefore, the map

$$
\mathcal{H o m}(\widetilde{A}, \widetilde{B}) \rightarrow \mathcal{H o m}(\widetilde{A}, B)
$$

is a Kan fibration. But the maps (6) and (5) coincide, so the condition (2) of 5.1 .4 is proven.

4.2.2. Fix now a cofibrant $\mathcal{O}$-algebra $A$ and denote $\mathfrak{g}=T_{A}$. Fix $(R, \mathfrak{m}) \in \operatorname{dgart} \leq 0(k)$.

Define a map of simplicial categories

$$
\alpha: \Gamma_{\mathfrak{g}}(R) \rightarrow W
$$

as follows. Let $z \in \mathrm{MC}(\mathfrak{m} \otimes \mathfrak{g})=\mathrm{Ob} \Gamma_{\mathfrak{g}}(R)$. Put

$$
\alpha(z)=(R \otimes A, 1 \otimes d+z) .
$$

Now, any element $g \in G_{n}=\exp \left(\Omega_{n} \otimes \mathfrak{m} \otimes \mathfrak{g}\right)^{0}$ defines a graded automorphism of $\Omega_{n} \otimes R \otimes A$. This obviously defines an isomorphism of $R \otimes \mathcal{O}$-algebras

$$
(R \otimes A, 1 \otimes d+z) \longrightarrow(R \otimes A, 1 \otimes d+g(z)) .
$$

The map $\alpha: \Gamma_{\mathfrak{g}}(R) \rightarrow W$ identifies $\Gamma_{\mathfrak{g}}(R)$ with the fibre of $\pi: W \rightarrow \bar{W}$ at $A$. Since $\pi$ is a fibration, this is weakly equivalent to the homotopy fibre of $\pi$.

Now Theorem follows since the nerve functor $\mathcal{N}$ preserves fibrations and weak equivalences by 5.3 .2 .

\subsection{Concluding remarks.}


4.3.1. The formula (2) defines a deformation functor for operad algebras not necessarily concentrated in non-positive degrees.

The definition seems to be correct also in this case, in spite of the fact that $T_{A}$ does not govern deformations of $A$ in this case. It seems that one should find in this case a more clever way to define the tangent Lie algebra. It might consist of "tame" derivations of a cofibrant resolution $\widetilde{A}$ of $A$, the ones which behave well with respect to a filtration on $\widetilde{A}$.

4.3.2. One can define "hard" formal deformations of a $\mathcal{O}$-algebra $A$ which deform not only the algebra $A$ itself but also the base operad $\mathcal{O}$. Then the universal deformation of $(\mathcal{O}, A)$ would provide the tangent Lie algebra $T_{A}$ with a canonical extra structure.

\section{Appendix: Simplicial CATEGORIES}

In this Section we present a more or less standard information about simplicial categories. It includes the description 5.1.5 of a CMC structure on the category sCat of small simplicial categories. This structure is a slight generalization of the one described in [DK].

5.1. Weak equivalences and fibrations in sCat. Here we define a closed model category structure on the category sCat of simplicial categories.

5.1.1. (Co)limits. The category sCat admits arbitrary limits and colimits. Inverse limits in sCat are induced by inverse limits in Ens in the obvious sense.

The existence of inductive limits in sCat follows by a general abstract nonsense from the existence of inductive limits in Ens. Note that the functor sCat $\rightarrow$ Ens assigning to each simplicial category the set of its objects, commutes with inductive limits. The set of morphisms of an inductive limit is freely generated by the morphisms of all categories involved, modulo an obvious equivalence relation.

Note that the existence of direct limits in sCat allows one to mimic the procedure of "adding variables". We will single out the following cases.

Adding an object. Given $\mathcal{C} \in$ sCat, denote $\mathcal{C}\langle *\rangle$ the coproduct of $\mathcal{C}$ with the trivial one-object category $*$.

Adding an ingoing arrow. Given $\mathcal{C} \in$ sCat, $x \in \mathrm{Ob} \mathcal{C}$, one defines $\mathcal{C}\langle * \rightarrow x\rangle$ with the set of objects $\mathrm{Ob} \mathcal{C} \amalg\{*\}$ and the set of morphisms freely generated by $\mathcal{M o r} \mathcal{C}$ and by the map $* \rightarrow x$.

Adding an outgoing arrow. The category $\mathcal{C}\langle x \rightarrow *\rangle$ is defined similarly to the above.

Adding maps between objects Given $\mathcal{C} \in$ sCat, $x, y \in \mathrm{Ob} \mathcal{C}$ and a map $\alpha: \mathcal{H}_{\mathcal{C}}(x, y) \rightarrow H$ of simplicial sets, the simplicial category $\mathcal{C}\langle x, y ; \alpha\rangle$ has the same objects as $\mathcal{C}$. Its set of morphisms is freely generated by $\mathcal{M o r C}$ and by $H$.

\subsubsection{Define the functor}

$$
\pi_{0}: \text { sCat } \rightarrow \text { Cat }
$$

as follows. For $\mathcal{C} \in \mathrm{s}$ Cat the category $\pi_{0}(\mathcal{C})$ has the same objects as $\mathcal{C}$. For $x, y \in \mathrm{Ob} \pi_{0}(\mathcal{C})$ 


$$
\operatorname{Hom}_{\pi_{0}(\mathcal{C})}(x, y)=\pi_{0}\left(\operatorname{Hom}_{\mathcal{C}}(x, y)\right)
$$

5.1.3. Definition. A map $f: \mathcal{C} \rightarrow \mathcal{D}$ in sCat is called a weak equivalence if the following properties are satisfied.

(1) The map $\mathcal{N}\left(\pi_{0}(f)\right)$ is a weak equivalence of simplicial sets.

(2) For all $x, x^{\prime} \in \mathrm{ObC}$ the map $f: \mathcal{H o m}\left(x, x^{\prime}\right) \rightarrow \mathcal{H o m}\left(f x, f x^{\prime}\right)$ is a weak equivalence.

5.1.4. Definition. A map $f: \mathcal{C} \rightarrow \mathcal{D}$ in sCat is called a fibration if it satisfies the following properties

(1) the right lifting property (RLP) with respect to "adding an ingoing or an outgoing arrow"

$$
\mathcal{A} \rightarrow \mathcal{A}\langle * \rightarrow x\rangle, \mathcal{A} \rightarrow \mathcal{A}\langle x \rightarrow *\rangle
$$

(see 5.1.1).

(2) For all $x, x^{\prime} \in \mathrm{ObC}$ the map $f: \mathcal{H o m}\left(x, x^{\prime}\right) \rightarrow \mathcal{H o m}\left(f x, f x^{\prime}\right)$ is a Kan fibration. This is equivalent the the RLP with respect to all maps $\mathcal{A} \rightarrow \mathcal{A}\langle x, y ; \alpha\rangle$ where $\alpha$ is an acyclic fibration (see 5.1.1).

5.1.5. Theorem. The category sCat admits a CMC structure with weak equivalences described in 5.1.3 and fibrations as in 5.1.4.

5.1.6. An explicit description of different classes of morphisms in sCat is given below. The proof of the Theorem is standard. It is given in 5.2 .

5.1.7. A map $f: \mathcal{C} \rightarrow \mathcal{D}$ in sCat is called an acyclic fibration if it is simultaneously a weak equivalence and a fibration.

Lemma. A map $f: \mathcal{C} \rightarrow \mathcal{D}$ is an acyclic fibration iff the following conditions are satisfied.

(1) the map $\mathrm{Ob} f: \mathrm{ObC} \rightarrow \mathrm{Ob} \mathcal{D}$ is surjective. In other words, $f$ satisfies the $R L P$ with respect to "adding an object map" $\mathcal{A} \rightarrow \mathcal{A}\langle *\rangle$.

(2) For all $x, x^{\prime} \in \mathrm{ObC}$ the map $f: \mathcal{H o m}\left(x, x^{\prime}\right) \rightarrow \mathcal{H o m}\left(f x, f x^{\prime}\right)$ is an acyclic Kan fibration.

Proof. If $f$ satisfies (1), (2), it is clearly an acyclic fibration. In the other direction, suppose $f$ is an acyclic fibration. Then the property (2) is clear. We have only to check that Ob $f$ is surjective. Since $f$ satisfies the RLP with respect to ingoing and outgoing arrows, $\mathcal{D}$ is a disjoint union of the full subcategories, defined by the image of $\mathrm{Ob} f$ and by its complement. Since $\pi_{0}(f)$ is a weak equivalence, it induces a bijection of the connected components of $\mathcal{C}$ and $\mathcal{D}$ and this proves the claim. 
5.1.8. A map $f: \mathcal{C} \rightarrow \mathcal{D}$ will be called a standard cofibration if there is a collection of maps $f_{i}: \mathcal{C}_{i} \rightarrow \mathcal{C}_{i+1}, i \in \mathbb{N}$ such that $\mathcal{C}=\mathcal{C}_{0}, \mathcal{D}=\lim _{\rightarrow} \mathcal{C}_{i}$, and each $f_{i}$ is a coproduct of maps of one of the following two types:

(1) Adding an object $\mathcal{C}_{i} \rightarrow \mathcal{C}_{i}\langle *\rangle$;

(2) Adding maps between objects $\mathcal{C}_{i} \rightarrow \mathcal{C}_{i}\langle x, y ; \alpha\rangle$ with $\alpha$ injective.

By 5.1.7, standard cofibrations satisfy the LLP with respect to all acyclic fibrations.

5.1.9. A map $f: \mathcal{C} \rightarrow \mathcal{D}$ will be called a standard acyclic cofibration if there is a collection of maps $f_{i}: \mathcal{C}_{i} \rightarrow \mathcal{C}_{i+1}, i \in \mathbb{N}$ such that $\mathcal{C}=\mathcal{C}_{0}, \mathcal{D}=\lim _{\rightarrow} \mathcal{C}_{i}$, and each $f_{i}$ is a coproduct of maps of one of the following three types:

$(1+)$ Adding an ingoing arrow $\mathcal{C}_{i} \rightarrow \mathcal{C}_{i}\langle * \rightarrow x\rangle$;

(1-) Adding an outgoing arrow $\mathcal{C}_{i} \rightarrow \mathcal{C}_{i}\langle x \rightarrow *\rangle$;

(2) Adding maps between objects $\mathcal{C}_{i} \rightarrow \mathcal{C}_{i}\langle x, y ; \alpha\rangle$ with $\alpha$ acyclic cofibration.

By 5.1.4, standard acyclic cofibrations satisfy the LLP with respect to all fibrations.

5.1.10. The following description of cofibrations and of acyclic cofibrations results from the proof of Theorem 5.1.5.

Corollary. 1. Any cofibration in $\mathrm{sCat}$ is a retract of a standard cofibration.

2. Any acyclic cofibration in sCat is a retract of a standard acyclic cofibration.

5.2. Proof of Theorem 5.1.5. The axioms (CM 1), (CM 2), (CM 3), (CM 4)(ii) are immediately verified.

(CM 5)(ii) Let $f: X \rightarrow Y$ be a map in sCat. Adding objects to $X$, we can ensure that the map $f: \mathrm{Ob}(X) \rightarrow \mathrm{Ob}(Y)$ is surjective. Then, adding maps between objects, we can decompose $f$ into a standard cofibration followed by an acyclic fibration.

This implies, in particular, that any cofibration is a retract of a standard cofibration.

To check the axiom (CM 5)(i) we need the following

5.2.1. Lemma. Standard acyclic cofibrations are acyclic cofibrations.

Proof. It is enough to prove that a $\operatorname{map} \mathcal{C} \rightarrow \mathcal{D}$ is a weak equivalence when $\mathcal{D}$ is obtained from $\mathcal{C}$ by one of the following ways.

(1) adding a number of ingoing arrows;

(2) adding a number of outgoing arrows;

(3) adding (simultaneously) maps between objects $x_{i}$ and $y_{i}$ along acyclic cofibrations $\alpha_{i}$ : $\mathcal{H o m}\left(x_{i}, y_{i}\right) \rightarrow H_{i}$.

In the first two cases the map $\mathcal{C} \rightarrow \mathcal{D}$ is easily split by an acyclic fibration. 
The shortest way to get the result in the case (3) is to use Proposition 7.2 of [DK] which claims the existence of CMC structure on the category of simplicial categories having a fixed set of objects.

(CM 5)(i) Let $f: X \rightarrow Y$ be a map in sCat. Adding ingoing and outgoing arrows to $X$, we can ensure that the image of $\mathrm{Ob}(X)$ under $f$ consists of a number of connected components of $\mathrm{Ob}(Y)$. From now on we can suppose, without loss of generality, that $f$ is surjective on objects. Then for a decomposition $f=p \circ i$ it is enough to check that $p$ satisfies condition (2) of 5.1.4 to ensure $p$ is a fibration.

Now applying step by step the procedure of adding maps between objects $\mathcal{C} \rightarrow \mathcal{C}\langle x, y ; \alpha\rangle$ along acyclic cofibrations $\alpha$, we can construct a decomposition $f=p \circ i$ with $p$ fibration and $i$ a standard acyclic cofibration. According to Lemma 5.2.1, $i$ is an acyclic cofibration.

Now, applying the proof of (CM 5)(i) to any acyclic cofibration $f$, we deduce that $f$ is a retract of a standard acyclic cofibration.

(CM 4)(i) By definition, any standard acyclic cofibration satisfies LLP with respect to all fibrations. Any acyclic fibration is a retract of a standard acyclic fibration, and therefore satisfies as well LLP with respect to all fibrations.

Theorem is proven.

\subsection{Simplicial nerve.}

5.3.1. In what follows we identify Cat with the full subcategory of $\Delta^{0}$ Ens. Then every simplicial category (and even every $\mathcal{C} \in \Delta^{0} \mathrm{Cat}$ ) can be seen as a bisimplicial set; its diagonal will be called the nerve of $\mathcal{C}$ and will be denoted $\mathcal{N}(\mathcal{C})$. If $\mathcal{C}$ is a "usual" category, $\mathcal{N}(\mathcal{C})$ is its "usual" nerve.

The functor $\mathcal{N}:$ sCat $\rightarrow \Delta^{0}$ Ens admits a left adjoint functor

$$
\text { SCAT }: \Delta^{0} \text { Ens } \rightarrow \text { sCat }
$$

defined by the properties

$\operatorname{ObSCAT}\left(\Delta^{n}\right)=[n]=\{0, \ldots, n\} ;$

$\mathcal{M o r} \operatorname{SCAT}\left(\Delta^{n}\right)$ is freely generated by $a_{i} \in \mathcal{H o m}_{n}(i-1, i), i=1, \ldots, n$;

SCAT commutes with arbitrary colimits.

5.3.2. Proposition. The nerve functor $\mathcal{N}$ : sCat $\rightarrow \Delta^{0}$ Ens preserves weak equivalences, fibrations and cofibrations.

Proof. 1. To check that $\mathcal{N}$ preserves the fibrations, it is enough to prove that the adjoint functor SCAT preserves acyclic cofibrations. For this we have to check that SCAT transforms any map $\Lambda_{i}^{n} \rightarrow \Delta^{n}$ to an acyclic fibration. This is an easy exercise (one should consider the cases $n=1$ and $n>1$ separately). Note that the same reasoning (even easier!) proves that $\mathcal{N}$ preserves acyclic fibrations - this is because SCAT preserves cofibrations. 
2. It is clear that $\mathcal{N}(f)$ is a weak equivalence provided $f$ is a weak equivalence bijective on objects. To prove the general claim, we present $f$ as a composition of an acyclic fibration with an acyclic cofibration and therefore reduce the problem to the case $f$ is an acyclic cofibration. Using 5.1.10, we can suppose that $f$ is of one of the types $(1+),(1-),(2)$ of 5.1.9. The type (2) does not change the set of objects, so we have nothing to prove. The maps of types $(1+),(1-)$ split, and the splitting map is an acyclic fibration. This proves the claim.

3. The claim about cofibrations is obvious.

\section{REFERENCES}

[DK] W. Dwyer, D. Kan, Simplicial localizations on categories, J. Pure Appl. Algebra 17(1980), $267-284$.

[GM] Goldman, Millson,

[H1] V. Hinich, Descent of Deligne groupoids, IMRN, 1997

[H2] V. Hinich, Homological algebra of homotopy algebras, Comm. in algebra, 25(10)(1997), 3291-3323.

[H3] V. Hinich, DG coalgebras as formal stacks, Preprint math-ag/9812034

[L] D. Lehmann, Théorie homotopique des formes différentielles (d'après D. Sullivan), Astérisque, 45, 1977.

[Q] D. Quillen, Homotopy algebra, Lecture Note in math., 43.

Dept. of Mathematics, University of Haifa, Mount Carmel, Haifa 31905 Israel 